Karl Georg Zinn

\title{
Arbeit, Nachhaltigkeit und Beschäftigung
}

\begin{abstract}
Die Analyse der Zusammenhänge von nachhaltigem Wirtschaften einerseits und Wachstum, Beschäftigung und Strukturwandel andererseits steht im Mittelpunkt. Anhand jüngst publizierten makroökonomischen Szenarien sowie einem mesoökonomischen Beispiel aus dem Wohnungsbau werden die Wachstums- und Beschäftigungswirkungen ökologisch orientierten Interventionismus auch quantitativ umrissen. Die Integration der Nachhaltigkeitspolitik in den langfristigen Strukturwandel bietet die Möglichkeit, der säkularen Wachstumsabschwächung zumindest temporär entgegen zu wirken.
\end{abstract}

\section{Nachhaltigkeit: Heute wirtschaften für die künftige Menschheit!}

Nachhaltigkeit (sustainability, sustainable development) meint im sozialökonomischen Zusammenhang die Beschränkung heutigen Ressourcenverbrauchs zugunsten der Existenzgrundlagen künftiger Generationen. Die lebende Generation soll ihre Wirtschaftsweise darauf einstellen, dass die mondiale Umweltsituation, die Verfügbarkeit erschöpfbaren Ressourcen und die der kommenden Menschheit vererbten Bestände an Sachkapital und Wissen, insbesondere auch des technologischen Wissens, ihr die Möglichkeit belässt, mindestens einen gleichen, wenn nicht einen höheren Wohlstand zu erreichen wie die heute existierende Weltbevölkerung. Kurz gesagt, wir sollen nicht auf Kosten unserer Enkel, Urenkel und deren Enkel und Urenkel leben. Offenkundig handelt es sich um ein ethisches Postulat, denn die lebende Generation hat kein existenzielles Interesse, das eigene Wohlstandstandswachstum zugunsten der Nachkommen zu beschränken oder gar Wohlstandseinbußen hinzunehmen. Der Gegenwartsegoismus steht der Nachhaltigkeit entgegen, und „Zukunftssolidarität“ wird sich um so schwerer vermitteln lassen, als der Zeitgeist selbst der Solidarität unter den Lebenden widerstreitet.

Die begriffliche Erstbestimmung der Nachhaltigkeit verdankt sich dem BrundtlandReport von 1987. Allerdings finden sich die grundlegenden Überlegungen schon in vorhergehenden Berichten wie etwa dem der „Brandt-Kommission“ (Überleben sichern 1980), und sie lassen sich bis auf die erste Studie des Club of Rome von 1972 (Meadows 1972) zurück verfolgen. Der Brundtland-Report formuliert das Nachhaltigkeitskonzept aus einer erdumspannenden Perspektive, denn die Umweltzerstörungen und der Ressourcenverbrauch sind zwar den einzelnen Nationalstaaten ursächlich zurechenbar, aber die Folgen machen sich nicht nur weltweit bemerkbar, sondern Schadensverursacher und Geschädigte fallen oft in eklatantem Maße auseinander. Beispielsweise sind die Hauptenergieverbraucher, die reichen Länder, auch die größten $\mathrm{CO}_{2}$-Emittenten, aber die durch den Treibhauseffekt hervorgerufenen Klimaveränderungen und deren Auswirkungen (Überschwemmungen, Unwetter, Tro- 
ckenheit usw.) verteilen sich geographisch weitgehend unabhängig von den Standorten der Schadensverursacher.

Die „Weltbank“ (Bank for Reconstruction and Development) vertritt ein Konzept der nachhaltigen Entwicklung, das den kommenden Generationen die gleichen Handlungsmöglichkeiten (pro Kopf) belässt, wie sie die gegenwärtige Generation aufweist. Die „Weltbank“ operationalisiert diese Zielvorgabe durch Quantifizierung der verschiedenen Kapitalbestände, d. h. von Sachkapital, Humankapital, Naturvermögen und Sozialvermögen(Fues 1996; Serageldin, 1996). Das Naturvermögen umfasst vor allem Land, Wasser, Wald und die Rohstoffe der Erdkruste. Die von der Weltbank für 192 Länder vorgelegte Kapitalbestandsrechnung ergibt eine von der Rangfolge nach den Pro-Kopf-Einkommen deutlich verschiedene Länderfolge. Erstaunlich ist allerdings die relativ einheitliche Struktur der Kapitalzusammensetzungen. Der Sachkapitalbestand weist mit 16-20\% den niedrigsten, der Humankapitalstock in der Regel den höchsten Anteil auf. Zwischen dem Humankapitalbestand eines Landes und seiner relativen Wohlstandsposition besteht ein enger Zusammenhang. Die 29 reichsten Volkswirtschaften verfügen zusammen über etwa zwei Drittel des globalen Humankapitalbestandes. In Deutschland erreicht der Humankapitalbestand ca. 80\% des Gesamtvermögens und liegt damit über dem entsprechenden US-Wert. Die USA weisen hingegen ein weit höheres Naturvermögen auf. Eine Substitution der Kapitalarten untereinander ist in nur in Grenzen möglich, so dass auch vom technischen Fortschritt nur beschränkte Hilfe gegen den (übermäßigen) Naturverbrauch zu erwarten ist: Schwimmende Fischfabriken gibt es genug, es fehlen die Fische (Fues 1996, 301). Zudem hängt die Richtung des technischen Fortschritts wesentlich von den Produktionsverhältnissen ab. Unter kapitalistischen Bedingungen wird der technische Fortschritt in der Regel auf Produktivitätssteigerungen nur bei jenen Ressourcen bzw. Produktionsfaktoren hin kanalisiert, die private Kosten verursachen. Wo keine Kosten anfallen, gibt es auch keinen gewinnwirtschaftlichen Grund zur Rationalisierung. Höhere Energie-, Umwelt- und generell höhere Ressourcenproduktivität lässt sich somit nur dann unter gewinnwirtschaftlichen Bedingungen erreichen, wenn die externen Kosten internalisiert werden. Dies setzt entsprechende staatliche Interventionen voraus. Auch bei den Produktinnovationen ist das Profitkriterium entscheidend. Beispielsweise besteht für die pharmazeutische Industrie kein Anreiz, Medikamente gegen Krankheiten zu entwickeln, die nur oder vorwiegend von kaufkraftschwachen Regionen bzw. Gesellschaftsschichten benötigt werden.

Die Nachhaltigkeitspolitik muss auf internationalen Vereinbarungen beruhen, die der Diskrepanz zwischen der geographischen Verteilung der Verursacher- und der Betroffenenstandorte Rechnung trägt. Die internationale Koordination ist aber auch notwendig, um die aus der Nachhaltigkeitspolitik und der damit verbundenen strukturellen Anpassungen resultierenden Kostenbelastungen möglichst wettbewerbsneutral zu gestalten. Nachhaltigkeitspolitik muss zwar durch die nationalen Volkswirtschaften umgesetzt werden, aber gerade deshalb bedarf es internationaler Abstimmung, um zwischenstaatliche Verzerrungen der Wettbewerbsfähigkeit in erträglichen Grenzen zu halten. Nationale Alleingänge in der Nachhaltigkeitspolitik sind jedenfalls nur in bescheidenem Umfang möglich, auch wenn sie ihrer Vorbildfunktion wegen durchaus praktische Bedeutung haben. Die Umweltkonferenz von Rio de Janeiro 1992 und die Anschlusskonferenzen folgten diesem Grundprinzip, mondiale Nachhaltigkeit auf dem Weg koordinierten nationalstaatlichen Handelns zu entsprechen. So wurde beispielsweise für die $\mathrm{CO}_{2}$-Emission auf der Kyoto-Konferenz (1997) von den Industrieländern ein quantifiziertes internationales Reduktionsziel akzeptiert und auf länderspezifische Beiträge aufgeteilt. In den Jahren 2008 bis 2012 soll die $\mathrm{CO}_{2}$-Emission der 
Industrieländer gegenüber der von 1990 deutlich vermindert werden. Dabei entfällt auf die Europäische Union eine Reduktion von -8\%, auf die USA -7\% und Japan -6\%. Einzelnen Ländern (u.a. Australien, Island und Norwegen) wurden noch gewisse Steigerungen zugestanden. Die nationale Umsetzung orientiert sich an den länderspezifischen Gegebenheiten, so dass eine Abstimmung von Nachhaltigkeitspolitik und den jeweils vordringlichen sozialökonomischen Aufgaben - in der Europäischen Union vor allem dem Abbau der Massenarbeitslosigkeit - möglich bleibt. Nach dem Scheitern der Den Haager Klimakonferenz im November 2000 haben sich die Chancen für ein international abgestimmtes und faires Verhalten im Umweltschutz wieder arg verschlechtert.

\section{$2 \quad$ Nachhaltigkeit ist mehr als Umweltschutz}

Nachhaltigkeitspolitik wird in der breiteren Öffentlichkeit vorwiegend mit Umweltschutz in Verbindung gebracht und als ein quasi isoliertes, von anderen Politikbereichen getrenntes Problem- und Handlungsfeld gesehen. Diese Deutung ist aus zweifachem Grund inadäquat. Erstens betrifft das Nachhaltigkeitsproblem die Grundprinzipien unserer Wirtschaftsweise und die Rahmenbedingungen der Wirtschaftsordnung. Solange Wirtschaftswachstum faktisch oberste Priorität genießt, bleibt der permanente Widerspruch zwischen Nachhaltigkeit und Wirtschaften bestehen. Zweitens erfordert die Umsetzung einer Nachhaltigkeitsstrategie den Einsatz des gesamten wirtschaftspolitischen Instrumentariums. Steuer- bzw. Finanzpolitik, Industrie- und Strukturpolitik, Außenwirtschaftspolitik usw. müssten auf die Nachhaltigkeitsziele orientiert werden, wenn diese erfolgreich realisiert werden sollen. Nicht zuletzt aber besteht eine sehr enge Verbindung zwischen Nachhaltigkeit und Wohlstandsverteilung - national und noch mehr international. Armut ist umweltschädlich (Gocht 1995), und Umweltzerstörungen sind häufig Ursachen nicht nur für Armut, sondern für schwere Menschenrechtsverletzungen (Menschliche Entwicklung 2000, 100 ff.). Der Globalisierungsprozess hat die Chancen nachhaltigen Wirtschaftens verschlechtert. Im Zuge der neoliberalistisch geprägten Globalisierung wird die Wirtschafts- und Gesellschaftspolitik auf die Ansprüche konkurrenzwirtschaftlicher Kapitalverwertung zugespitzt: „Das Deregulierungsinteresse annulliert... das Schutzinteresse“ (Sachs 2000a, 979).

Bei der Nachhaltigkeitspolitik geht es hingegen um die Verknüpfung von drei Problembereichen: dem ökologischen, dem sozialen und dem wirtschaftlichen. Dieses erweiterte Verständnis von Nachhaltigkeit wurde jüngst in einem Verbundprojekt zur Nachhaltigkeitsforschung (HBS, 2000) umrissen. Die dort formulierte ,aktuelle“ Fassung des Nachhaltigkeitskonzepts

- „geht von einer gleichrangigen Berïcksichtigung der drei Nachhaltigkeitsdimensionen aus;

- räumt Fragen der sozialen Integration und Gerechtigkeit eine große Bedeutung ein;

- gibt der Verringerung der Arbeitslosigkeit einen größeren Stellenwert;

- sieht die Rolle des Staates in Relation zu anderen Akteursgruppen;

- baut auf interdisziplinären Ansätzen auf;

- gibt der Kommunikation mit gesellschaftlichen Gruppen und Organisationen einen hohen Stellenwert" (HBS 2000, 7)

Die Einbeziehung des Beschäftigungsproblems in die Nachhaltigkeitsstrategie erscheint insofern von entscheidender Bedeutung zu sein, als sich hieran der Konflikt zwischen den 
Interessen an Arbeitsplatz- bzw. Einkommenssicherheit der lebenden Generation und den Zukunftserfordernissen am schärfsten abzeichnet. Nachhaltigkeitspolitik, die zulasten der Beschäftigung geht, trifft auf breite Widerstände. Sicherlich besteht ein enger Zusammenhang zwischen dem ungelösten Beschäftigungsproblem und dem stark rückläufigen Interesse der deutschen Öffentlichkeit an Umweltproblemen. Der Einnahme- und Mitgliederverlust der deutschen Greenpeace-Organisation während der jüngeren Vergangenheit ist hierfür nur ein Beleg unter vielen (Bode, 2000). Es liegt auf der Hand, dass eine opportunistische Regierung bei fehlendem öffentlichen Meinungsdruck auch nicht motiviert ist, sich einer konsequenten Nachhaltigkeitspolitik zu verschreiben. Rhetorik und Handeln klaffen auseinander. Beispielhaft sei auf die unzulängliche Umsetzung der Klimaschutzpolitik durch die deutsche Bundesregierung verwiesen. Auf der Berliner Klimaschutzkonferenz 1995 wurde von der deutschen Regierung zugesagt, die bis zum Jahr 2005 um $25 \%$ gegenüber dem Stand von 1990 zu senken. Weder die Regierung Kohl noch die rot-grüne Regierung haben die erforderlichen - und technisch möglichen - Umsetzungsmaßnahmen ergriffen. Im Sinn der neoliberalistischen Grundorientierung der Schröder-Regierung wurde sogar die für eine Ausschöpfung der technisch-wirtschaftlichen Reduktionspotentiale unabdingbare, zu Beginn der rot-grünen Koalition noch avisierte staatliche Eingriffsintensität zugunsten ,,marktwirtschaftlichen“" Vorgehens wieder verworfen (Ziesing 2000, $521 \mathrm{f}$.).

\section{Wachstum und Nachhaltigkeit}

Der grundsätzliche Widerspruch zwischen Wachstum und Nachhaltigkeit lässt sich auf längere Sicht nur durch Wachstumsreduktion lösen. Denn jede Art von Wachstum verbraucht (natürliche) Ressourcen. Dieser langfristige Widerspruch wird auch nicht durch höhere Ressourcenproduktivität aufgehoben. Denn wenn die Nachhaltigkeitsvorteile höherer Umwelt- und Energieeffizienz durch Mengenwachstum überkompensiert werden - geringerer Kraftstoffverbrauch pro Kilometer, aber es wird mehr gefahren - bleiben die notwendigen Ressourceneinsparungen aus. Höhere Ressourcenproduktivitäten bremsen den Kosten- bzw. Preisanstieg, so dass unter Umständen sogar Verbrauchssteigerungen begünstigt werden. Es wird also darauf ankommen, einerseits die technisch-wirtschaftlichen Produktivitätspotentiale beim Ressourceneinsatz voll auszuschöpfen, andererseits aber durch Wachstumsbegrenzung dafür zu sorgen, dass es auch tatsächlich zu insgesamt verminderten Verbräuchen kommt.

Die Plausibilität von Wachstumsreduktion bis zu völliger Stagnation in reichen Volkswirtschaften wird gegenwärtig in der Öffentlichkeit nicht mehr erkannt, ja kaum noch erörtert. Die Aufklärungseffekte der früheren Diskussion über die „Grenzen des Wachstums“, über „qualitatives Wachstum“, über die Unsinnigkeit des Wegwerf- und Verschleißkonsums und der künstlichen Produktveralterung mit den ihr korrespondierenden Pseudoinnovationen, der Diskriminierung langlebiger Güter etc. wurden von anderen Themen überlagert. In den wohlhabenden Volkswirtschaften entfällt ein Großteil der Konsumgüterproduktion auf reinen Luxus- und Prestigekonsum, aber selbst dort sind relative Sättigungserscheinungen nicht zu übersehen; die Nachfrage wird preis- und einkommensunelastischer. In den Wohlstandswirtschaften besteht im Hinblick auf die angemessene Versorgung der Gesamtbevölkerung längst kein dringender Wachstumsbedarf mehr, sondern die brennenden sozialökonomischen Probleme sind letztlich verteilungspolitischer Art. Die Wiederbelebung 
der Debatte um die richtige Wachstumsqualität bildet daher einen unabdingbaren Bestandteil einer erfolgversprechenden Nachhaltigkeitspolitik. „Qualitatives“Wachstum muss heute als Wachstum zugunsten der Umwelt verstanden werden: Umweltschutz als temporäre Wachstumskraft (Vinod 2000). Im folgenden Abschnitt wird hierauf näher einzugehen sein.

Die bisherige Entwicklung der Umwelt- und Energieproduktivität verlief ohnehin in Größenordnungen, die nicht ausreichen, um bei den erwarteten bzw. angestrebten Wachstumsraten des Bruttoinlandsprodukts von 2-3\% den Umwelt- und Energieverbrauch auch nur konstant zu halten, geschweige denn eine deutliche Reduktion zu erreichen. Der jährliche Anstieg der Energieproduktivität in Deutschland nach 1990 lag mit 2\% zwar deutlich über dem durchschnittlichen BIP-Wachstum der neunziger Jahre (1,5\%), aber diese BIP-Wachstumsrate gilt als viel zu niedrig, und es ist zu erwarten, dass das BIP-Wachstum im Mehrjahresdurchschnitt wieder über $2 \%$ steigen wird. Um das bundesdeutsche Ziel, die $\mathrm{CO}_{2}$ -Emissionen bis 2005 um 25\% unter das 1990er Niveau zu drücken, in etwa zu erreichen, müsste nach Berechnung des Deutschen Instituts für Wirtschaftsforschung ein jährlicher Anstieg der Energieproduktivität von über 3\% realisiert werden (Ziesing 2000, 524). Technisch-wirtschaftlich wären solche Produktivitätssteigerungen zwar möglich, aber sie erforderten einen erheblichen staatlichen Interventionsaufwand, denn die zur Ressourceneinsparung bzw. Steigerung der Ressourcenproduktivität erforderlichen Investitionen erstrecken sich über die gesamtwirtschaftliche Breite und beträfen zu einem erheblichen Teil auch private Haushalte, also „Kleininvestoren“. Energieeinsparung im Wohnbereich und beim privaten Kraftfahrzeugverkehr sind in größerem Umfang nur durch besondere Anreize bzw. Fördermaßnahmen zu erreichen (Bartholomai 2000; Ziesing 2000), d. h. es gibt keine „rein“ marktwirtschaftlichen Lösungen, sondern staatliche Vorgaben und Einflussnahme sind unabdingbar.

\section{Beschäftigungspotentiale einer integrierten Nachhaltigkeitspolitik}

Der langfristige Widerspruch zwischen Nachhaltigkeit und Wachstum gilt nicht in gleicher Weise auf kurze bis mittlere Sicht. Wenn nämlich die unausgeschöpften Produktionskapazitäten und die unbeschäftigten Arbeitskräfte zugunsten von Umweltschutzmaßnahmen, somit auf der Linie einer Nachhaltigkeitsstrategie genutzt werden, so ergeben sich daraus gleichzeitig Wachstum und Beschäftigungszunahmen. Es geht also um ein qualitativ zielorientiertes Wachstum. Dies bedeutet, erwünschte Produktionsbereiche zu fördern, unerwünschte, weil umweltschädigende einzuschränken. Solch ein reguliertes Wachstum erfordert ein erhebliches Maß an staatlichen Interventionen und bedeutet damit auch eine lenkende Einflussnahme auf die Investitionen. Ob dabei vorwiegend indirekte, über Anreize, Nachfragestimulierung und dergleichen ,marktkonforme“ Maßnahmen wirksame Instrumente eingesetzt werden oder aber direkte, auf Gebote und Verbote hinauslaufende Eingriffe vorgenommen werden, sollte pragmatisch entschieden werden. Allerdings ist streng darauf zu achten, dass nicht unter dem opportunistischen Vorwand, „marktkonform“ handeln zu wollen, bloß symbolische Politik betrieben wird und die Nachhaltigkeitsziele verfehlt werden. Kurz gefasst, lässt sich die skizzierte Handlungsstrategie auf die Faustformel bringen, „Umweltschutz zugunsten von Wachstum und Beschäftigung “. In gewisser Weise handelt es sich um ein riesiges Wiederaufbauprogramm - nach einem über zweihundertjäh- 
rigen Krieg der industrialisierten Wohlstandsgesellschaften gegen Umwelt und Natur.

Der Beschäftigungsbeitrag des Umweltschutzes liegt in Deutschland mindestens bei einer Million Arbeitsplätzen. Die sektoralen Beschäftigungseffekte der Umweltpolitik werden für 1994 auf insgesamt 955900 Erwerbstätige beziffert; das waren 2,7 \% der Gesamtbeschäftigung (vgl. Tabelle 1).

Tab. 1: Sektorale Beschäftigungseffekte der Umweltpolitik 1994

Quelle: Projektgemeinschaft DIW/ifo/IWH/RWI 1996; hier zitiert nach: Blien u.a., 2000, 130

Umweltbezogene Produktion lässt sich grob zwei Zielsetzungen zuordnen: der Sanierungspolitik und dem vorbeugenden Umweltschutz. Sanierungsmaßnahmen betreffen die Erneuerung bzw. den Austausch vorhandenen Sachkapitals im weiten Sinn. So würde etwa die Erneuerung der in vielen Städten völlig veralteten und lecken Versorgungs- und Entsorgungsleitungen den natürlichen Wasserhaushalt entlasten. Vorbeugender Umweltschutz umfasst Maßnahmen, die umweltbelastende Produktions- und Konsumaktivitäten reduzieren oder auch völlig unterbinden. Vorbeugender Umweltschutz erfordert meist strukturelle Umstellungen. Wenn etwa der Anteil fossiler Energieerzeugung gesenkt werden soll oder eine Verlagerung vom Straßenverkehr auf Schiene und Wasserwege angestrebt wird, so impliziert das nicht nur entsprechende Investitionen, sondern relativ weitreichende Verhaltens- und Einstellungsänderungen. Den umweltschädigenden „Geschwindigkeitswahn“ zugunsten einer Entschleunigung zu kurieren, birgt zwar erhebliche Vorteile für eine schonendere Umweltnutzung, aber solche bis in die Tiefen der etablierten Wirtschaftsmentalität reichenden Veränderungen treffen auf unvergleichlich höhere Barrieren als die herkömmlichen Maßnahmen des sanierenden Umweltschutzes. Sanierung und Vorbeugung können je nach Einzelfall durch nachsorgende, additive Technik (end-of-the-pipe-Technik) oder integrierten Umweltschutz erfolgen. Bisher dominiert - entgegen landläufigen Erwartungen - in Deutschland ganz eindeutig die erstgenannte Technik (Blien u.a. 2000, 131 f.). Dies erscheint 
ökonomisch plausibel, da diese Technik die vorhandenen Produktionsanlagen ergänzt, aber im Übrigen einsatzfähig belässt, während integrierter Umweltschutz i. d. R. die Verschrottung der alten Anlagen erfordert und höhere Innovationsanforderungen und -risiken aufweist.

Zwei Beispiele aus der jüngeren Literatur sollen näher verdeutlichen, welche Wachstumsund Beschäftigungspotentiale im Umweltschutz enthalten sind.

\subsection{Der Status quo ist auch für die Beschäftigung schlechter}

In dem bereits erwähnten Verbundprojekt „Arbeit und Ökologie“1 (BS, 2000) werden drei Szenarien gegenüber gestellt und neben anderen Indikatoren die jeweiligen Wachstums-, Produktivitäts- und Beschäftigungsentwicklungen bis 2020 umrissen. Die „ökonomischsoziale Reformstrategie " betont den traditionellen Ansatz, Beschäftigung durch Wachstum zu erreichen. Jedoch soll durch Umweltschutzabgaben (Klima, Luft, Verkehr, Gewässer, Boden), die über einen Zeitraum von zehn Jahren jährlich ansteigen, eine Reduktion der umweltbelastenden Emissionen erreicht werden. - Das ,ökologisch-soziale Szenario “ sieht ein umfassenderes Instrumentarium der Umweltpolitik vor, das neben indirekten Maßnahmen auch direkte Vorgaben bzw. Beschränkungen (z. B. Tempolimit) beinhaltet. Diesen beiden Veränderungsszenarien wird als Kontrastmodell die Fortschreibung des Status quo, d.h. ,Kostenentlastung durch niedrige Löhne und weniger Staat“ gegenüber gestellt. In Tab. 2 sind einige Berechnungsergebnisse der Szenarien wiedergegeben.

Tab. 2: Ausgewählte Ergebnisse der Szenarien des „Verbundprojekts“ für das Jahr 2020

Quelle: HBS, 2000

Diese Angaben dort entsprechen Plausibilitätserwartungen mit der Ausnahme des Bruttoinlandsprodukts. Das BIP-Wachstum fällt in den beiden „ökologischen“ Szenarien deutlich höher aus als im Kontrastszenario der Status-quo-Prognose. Der Gesamtanstieg des BIP zwischen 1994 und 2020 beträgt beim ökonomisch-sozialen Szenario 75\%; bei ökologischsozialer Strategie 62\% und bei Status-quo-Verlauf nur 51\%. Die Erklärung für dieses bei oberflächlicher Betrachtung überraschend schlechte Wachstumsergebnis der Status-quoAlternative wird verständlich, wenn die eigentlichen Ursachen für die (deutsche) Wachstumsschwäche in den Blick gerückt werden. Es mangelt nämlich seit langem an der für eine

1 Es handelt sich um eine von der Hans-Böckler-Stiftung als Projekträgerin initiierte Studie, an der das Deutsche Institut für Wirtschaftsforschung, Berlin, das Wuppertal Institut für Klima, Umwelt, Energie und das Wissenschaftszentrum Berlin beteiligt sind. 
deutliche Beschäftigungsexpansion erforderlichen Binnenmarktnachfrage. Der Absatz herkömmlicher Konsumgüter - selbst im Luxusbereich - trifft auf relative Sättigung, d. h. die Preiselastizität der Nachfrage liegt deutlich niedriger als früher und scheint weiter zu sinken. Umweltschutz gehört hingegen zu den Produktionen, bei denen noch längst keine relative Sättigung erreicht ist. ${ }^{2}$ Die deutsche - und generell die westeuropäische - Wachstumsschwäche der vergangenen beiden Jahrzehnte findet in der Keynesschen Stagnationstheorie eine überzeugende Erklärung (Zinn 1999, 1998). Sowohl das ökonomisch-soziale als auch das ökologisch-soziale Szenario impliziert eine klare nachfragepolitische Strategie. Allerdings erhält beim ökonomisch-sozialen Ansatz die Nachfrage- und Umverteilungskomponente stärkeres Gewicht. Beim ökologisch-sozialen Konzept kommt hingegen der Arbeitszeitverkürzung (um 20\%, so dass bis zum Jahr 2020 die 30-Stunden-Woche erreicht wird) zentrale Bedeutung - für die Beschäftigungspolitik - zu.

\subsection{Arbeitsplätze durch Energieeinsparung im Wohnbereich}

Reformszenarien machen es ihren Gegnern relativ leicht, mit Verweis auf die Zukunftsunsicherheiten und die unvermeidlich voluntaristische Prämissensetzung den gesamten Ansatz in Frage und unter „Utopieverdacht“ zu stellen. Solcher zur Pauschalierung tendierender Ablehnung sind die auf einzelne Branchen und Sektoren bezogenen partiellen Untersuchungen weit weniger ausgesetzt. Sie können zwar keine gesamtwirtschaftliche Nachhaltigkeitsstrategie entwerfen, wohl aber zeigen sie, wie sich das Grundprinzip der Nachhaltigkeit im Detail umsetzen lässt, und ob und wie daraus Wachstums- und Beschäftigungsgewinne resultieren. Zudem können solche Detailstudien gegebenenfalls die Generallinie eines Szenarios bestätigen - oder auch widerlegen. Im Folgenden sei beispielhaft auf die Untersuchung des Deutschen Instituts für Wirtschaftsforschung zur Energieeinsparung im Wohnungsbau eingegangen (Bartholomai 2000).

Der Anteil der deutschen Haushalte am gesamten Endenergieverbrauch (ohne Verkehr) ist zwischen 1990 und 1998 von 25,1 auf 29,6\% gestiegen. Entsprechend nahmen auch die auf die Haushalte entfallenden $\mathrm{CO}_{2}$-Emissionen zu - von 128 Mio. 1990 auf 136 Mio. 1998 (Bartholomai 2000, 500). In den neunziger Jahre wurden zwar verschiedene Förderprogramme für wohnungsbezogene Energiesparmaßnahmen aufgelegt, aber - wie aus der DIW-Studie hervorgeht - war deren (Anreiz)Wirkung relativ gering. Insbesondere konzentrierten sich die Maßnahmen auf den Mietwohnungsbereich, und die relativ hohen Einsparungspotentiale bei Eigenheimen wurden in den Fördermaßnahmen nur sehr unzulänglich berücksichtigt. Hier ergibt sich - bei veränderter bzw. erweiterter Förderung - ein erhebliches Einsparungspotential an Kohlendioxyd, und zugleich könnte ein relativ starker Wachstums- und Beschäftigungseffekt ausgelöst werden. Die DIW-Berechungen beziffern das durch entsprechende Förderung direkt auslösbare Volumen der energiesparenden Investitionen allein bei westdeutschen Eigenheimen und Eigentumswohnungen auf jährlich 9 Mrd. Mark. Ergänzend könnte im Mietwohnbereich noch eine Investitionssumme von $1 \mathrm{Mrd}$. ausgelöst werden.

2 „Die Beschäftigungsentwicklung wird gefördert, wenn jenen Branchen der Volkswirtschaft günstigere Bedingungen eingeräumt werden, in denen einerseits mit aufnahmefähigen Märkten, andererseits mit schnellem technischen Fortschritt zu rechnen ist. Überlegt man, welche Branchen dies im Fall der Bundesrepublik Deutschland sein werden, so finden sich nicht viele. Unter ihnen sind aber auf jeden Fall Umwelttechnik- und Umweltschutzdienstleistungen. Damit bietet diese Querschnittsbranche Aussichten nicht nur bei der Lösung von Umweltproblemen und bei Ansätzen zur nachhaltigen Entwicklung, sondern kann eine Perspektive zur Lösung des Beschäftigungsproblems anbieten“ (Blien u.a. 2000, 128). 
Dieses Investitionsvolumen von insgesamt 10 Mrd. würde über die Anstoßeffekte in den Vorleistungsbereichen sowie durch die steigende Konsumnachfrage, die infolge der zusätzlichen Beschäftigung eintreten wird (Multiplikatoreffekt), eine Zunahme des BIP von ca. 24 Mrd. Mark bewirken. „Die Zahl der zusätzlich Beschäftigten wäre (unter Berücksichtigung des Produktivitätsanstiegs) im Jahr 2005 mit gut 100000 zu veranschlagen“ (Bartholomai 2000, 506). Die vom Staat in Form von Mindersteuereinnahmen zu tragende Nettobelastung der Fördermaßnahmen wird auf 200 Mio. im Anfangsjahr und etwa 2 Mrd. Mark im letzten, zehnten Jahr beziffert (Bartholomai 2000, 505).

\section{$5 \quad$ Strukturwandel zugunsten nachhaltigen Wirtschaftens}

Im Unterschied zum extensiven Wachstum der vorindustriellen Epoche, das mangels merklicher Produktivitätszunahmen keinen wesentlichen Strukturwandel aufwies, begann mit der industriellen Revolution ein bis heute anhaltender Anstieg der Arbeitsproduktivität. Er ist die notwendige Voraussetzung intensiven Wachstums (= Anstieg des statistischen Pro-KopfEinkommens). Steigende Einkommen bzw. die damit einher gehende Zunahme des wirtschaftlichen Lebensstandards führen zu relativer Sättigung auf traditionellen Märkten (Stagnationsmärkte). Aus dem Zusammenspiel von produktivitätswirksamem technischen Fortschritt (Prozessinnovationen) und relativer Sättigung resultierten die (langfristigen) Anteilsverschiebungen von der Landwirtschaft zur Industrie und gegenwärtig zu den Dienstleistungen, wie sie Jean Fourastié in seiner „klassischen“ Analyse zum sektoralen Strukturwandel (Fourastié, 1954) nicht nur beschrieb, sondern mit Bezug auf die Dienstleistungsexpansion auch grundsätzlich zutreffend prognostizierte. Allerdings basierte der Zukunftsoptimismus Fourastiés, nämlich der Erwartung, dass der Dienstleistungssektor auch bei sinkenden Wachstumsraten des BIP ein Auffangbecken für alle in der Urproduktion und der Industrie entlassenen Arbeitskräfte bilden würde, u. a. auf drei von der Realität widerlegten Prämissen: erstens der Nichtrationalisierbarkeit von Dienstleistungen (keine Produktivitätszunahmen bei Diensten); zweitens einer gesellschaftlich ausgeglichenen Einkommensverteilung derart, dass alle (neuen) Dienstleistungsarbeitsplätze keine Lohneinbußen brächten, sondern die Masseneinkommen am gesamtwirtschaftlichen Produktivitätsniveau orientiert werden; drittens eine hohe Einkommenselastizität der Dienstleistungsnachfrage (,Hunger nach Tertiärem“), so dass steigende Einkommen auch zu höherer Dienstleistungsnachfrage führen würden - und nicht etwa in erheblichem Umfang in die Ersparnisbildung abflössen. Statt der Fourastiéschen „tertiären Zivilisation“ zeigen sich jedoch Tendenzen einer „tertiären Krise“ (Zinn 1997, 98 ff.).

Die für Vollbeschäftigungserfordernisse zu schwache Absorptionsfähigkeit des Dienstleistungssektors bedeutet jedoch keine (empirische) Widerlegung der Fourastiéschen Langfristprognose des Strukturwandels und der Stagnation. Vielmehr wurde seine Theorie von der jüngeren Wirtschaftsgeschichte vollauf bestätigt. Insbesondere vermochte der sekundäre Sektor, also die Industrie, trotz der hohen Innovationsdynamik, die keineswegs nur rationalisierungswirksam wurde, sondern auch eine Vielzahl neuer Produkte - insbesondere auf der Grundlage der „mikroelektronischen Revolution“ - hervorbrachte ihrem prognostizierten Schicksal eines fortlaufenden Anteilsverlustes am BIP und einem absoluten Rückgang der Beschäftigung nicht zu entgehen. 
Wie bereits dargelegt tritt der Strukturwandel als Folge des intensiven Wachstums ein. Der Wachstumsprozess hält jedoch nur so lange an, als den Stagnations- und Schrumpfungsbereichen kompensierende Wachstumsbranchen folgen. Die orthodoxe Vorstellung, es ließen sich durch Produktinnovationen immer wieder genügend Wachstumsfelder erschließen, um den wiederkehrenden Sättigungsprozessen auf mehr und mehr Märkten entgegen zu wirken, erwies sich jedoch als Irrtum. Es gelang eben nicht, durch die technologisch brillianten Produktinnovationen der Mikroelektronik die relative Schrumpfung des sekundären Sektors aufzuhalten. Die Mikroelektronik hat aber durch ihr auf den Dienstleistungsbereich ausgreifendes Rationalisierungspotential auch die „große Hoffnung“ enttäuscht, die sich auf die Beschäftigungsexpansion im Dienstleistungssektor richtete. Große Teile des Dienstleistungsbereichs sind infolge ihres Produktivitätswachstums dem gleichen längerfristigen Sättigungsprozess unterworfen wie die Vielzahl der Industriegütermärkte. Der Umweltbereich bzw. eine auf Nachhaltigkeit ausgerichtete Wirtschafts- und Gesellschaftspolitik böte einen neuen Wachstumsmarkt, dessen Absatz- und damit Produktions- und Beschäftigungspotential durchaus dem einer expansiven Rüstungsökonomie nicht nachstünde. Wie bei der Rüstungsexpansion handelt es sich beim Umweltschutz jedoch nicht um einen marktwirtschaftlichen Selbstläufer, sondern der Staat muss als zentraler Akteur den Expansionsprozess in Gang bringen. Beim Umweltschutz ist es - anders als bei der Rüstung - nicht erforderlich, dass der Staat selbst als Nachfrager auftritt, sondern es genügt, über Anreiz- und Gebotsmaßnahmen, private (Umweltschutz-)Nachfrage zu stimulieren. Das kleine Beispiel der staatlich geförderten Nachfrage nach dem geregelten Kfz-Katalysator zeigt, wie das Grundprinzip funktioniert. Es geht also um administrativ bzw. interventionistisch erzeugte Endnachfrage, die dann Investitionen und Innovationen induziert.

Zum besseren Verständnis der Entstehung relativer Marktsättigung, des fundamentalen Nachfrageproblems hoch entwickelter Volkswirtschaften, sei nachfolgend kurz skizziert, wie sich Produktivitätssteigerungen auf die Beschäftigungsentwicklung in einer Branche auswirken: Zu Beginn des Kosten senkenden Rationalisierungsprozesses ist - unter der realistischen Voraussetzung einer hohen Preiselastizität der Nachfrage (= ungesättigter Markt) - mit starkem Nachfragewachstum zu rechnen, das zu einer erheblichen Produktionsund meist auch Beschäftigungsausweitung führt. Doch mit zunehmender Marktsättigung überwiegen bei anhaltendem Produktivitätsfortschritt die Beschäftigung senkenden Effekte der Rationalisierung (vgl. Abbildung 1). Die zu Beginn der Entwicklung hohe Preiselastizität der Nachfrage bleibt eben infolge der relativen Sättigung nicht erhalten, sondern Absatz und somit auch die Produktion stagnieren, die Beschäftigung sinkt rationalisierungsbedingt (Blien u. a. 2000; Zinn 1999; Schettkat 1997). Wenn die durch Rationalisierung bedingten Stückkosten- bzw. Preissenkungen wegen der geringen Preiselastizität der Nachfrage kein Absatz-, Produktions- und Beschäftigungswachstum mehr hervorrufen, so ist das um so weniger durch Lohnsenkungen zu erreichen. Denn sie verstärken infolge sinkender Massenkaufkraft noch die relative Nachfrageschwäche. 


\section{Abb.1: Beschäftigung der Branche $X$ in Abhängigkeit von der Nachfrageentwicklung bei anhaltende Produktivitätswachstums*}

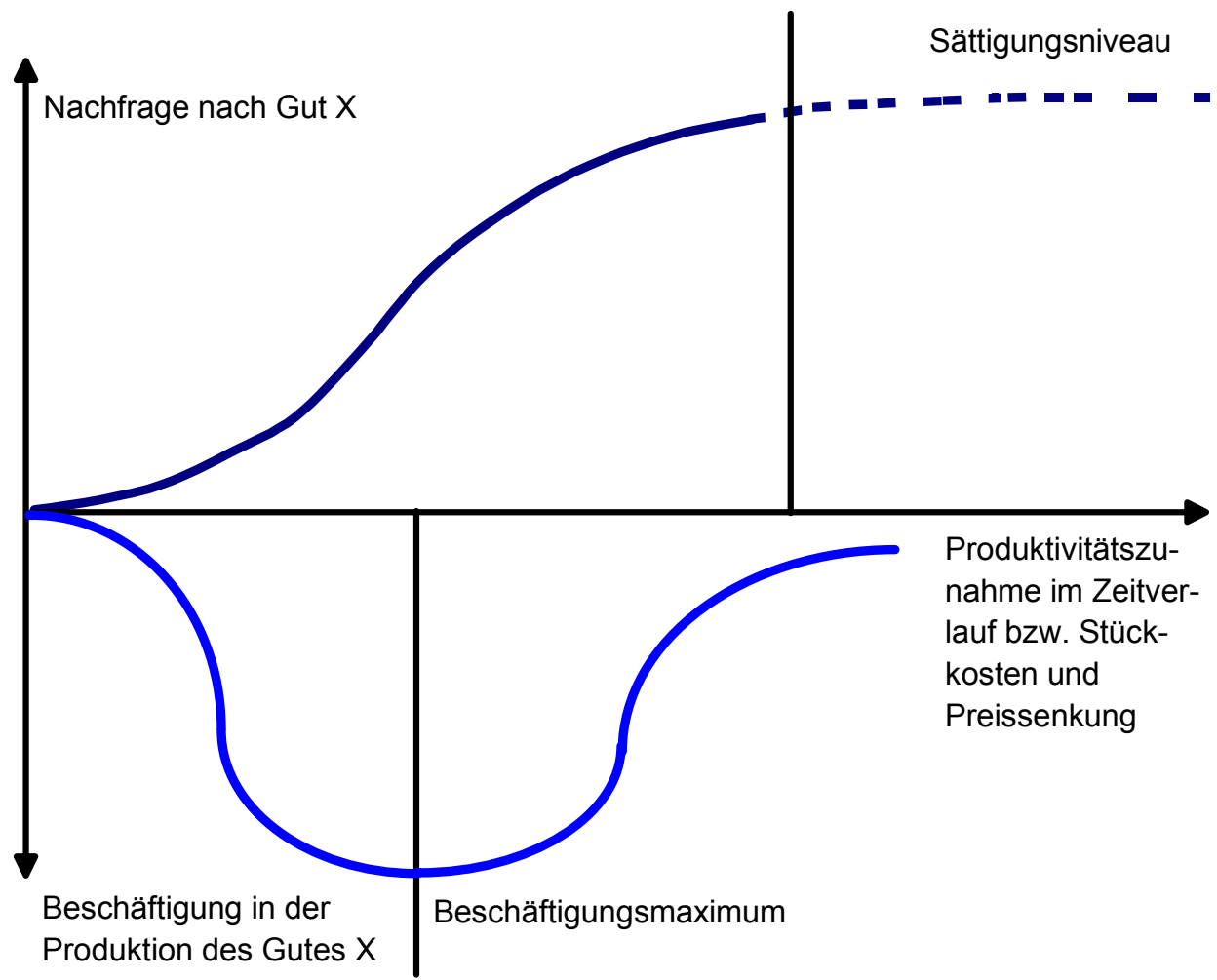

Quelle: Zinn, 1999, 4; * Stückkostenreduktion

Die in der Stagnationsbranche entstehende ,technologische Arbeitslosigkeit“ kann nur auf zwei Wegen wieder abgebaut werden: durch Nachfragewachstum, was entsprechende Wachstumsmärkte voraussetzt, oder mittels Arbeitszeitverkürzung. Das ökonomisch-soziale Szenario des dargelegten Verbundprojekts betont die Nachfragepolitik, das ökologischsoziale die arbeitszeitpolitischen Maßnahmen, um mehr Beschäftigung zu erreichen. Das Nachhaltigkeitskonzept bietet nun die Möglichkeit, neue Wachstumsbereiche zugunsten von Umweltschutz und ökologisch verträglicher Produktion zu schaffen (Blien u.a. 2000). Die während der Vergangenheit kumulierten Umweltzerstörungen begründen einen kaum abschätzbaren Sanierungsbedarf. Das zugunsten der Umweltsanierung mögliche Wachstum müsste selbst wiederum ökologischen Nachhaltigkeitskriterien genügen. Denn es wäre ein Schildbürgerstreich, die alten Umweltschäden zu beseitigen und dabei neue zu verursachen.

Sanierung und vorbeugender Umweltschutz erfordern für eine mehr oder weniger lange Übergangszeit die Produktion von entsprechenden Ausrüstungen und Anlagen, also Sachinvestitionen. Diese Nachfrage ist in erheblichem Umfang auf den sekundären Sektor, die 
Industrie, gerichtet. Industrielle Produktion ist jedoch mit weiterem Ressourcenverzehr verbunden, d. h. es kommt zu keiner grundsätzlichen Abkoppelung von Produktion und Natur- und Energieverbrauch i. w. S. Die Komplementarität von Arbeit und Naturstoffen wird nicht aufgelöst. Auf längere Sicht wird es jedoch darauf ankommen Arbeit weitgehend vom Naturverbrauch zu lösen. Dies ist auch der Fourastiéschen Vision einer ,tertiären Zivilisation“ impliziert: Arbeit löst sich weitgehend von der Komplementarität zu anderen Faktoren und wird mehr zu einer tertiären Leistung per se. Als zwei auch beschäftigungspolitisch bedeutsame Beispiele seien Pflegedienste und Bildungsaktivitäten genannt. Sie können zwar auch stoffaufwändig betrieben werden, aber die Anreicherung solcher Dienstleistungen mit kostspieligen, materialintensiven Apparaturen dürfte häufig mehr dem Marketing der Anbieter solcher Gerätschaften geschuldet sein als tatsächlichen Arbeitserleichterungen. Ähnliches gilt für die Aktivitäten einer „Freizeitgesellschaft“. Freizeit lässt sich recht umweltschädlich und konsumintensiv gestalten (z. B. Fernreisen) oder aber auch fast kostenlos und ohne Umweltbelastungen (Lektüre, Spaziergänge, Radfahren - vom off-roadFanatismus abgesehen).

Wie schon bemerkt wurde, kann Nachhaltigkeit auf Dauer nicht ohne Veränderung der Verhaltensweisen und der ihnen zugrunde liegenden Mentalitäten gelingen. Das Nachhaltigkeitsproblem erfordert eine „Suffizienzrevolution“, und das würde auch weitreichende Veränderungen im Arbeitsleben und im Konsumverhalten mit sich bringen (müssen).

\section{Literatur}

DIW (10/1999): Energieverbrauch im Verkehr in Deutschland. Verringerung der Wachstumsraten in den neunziger Jahren; in: DIW-Wochenbericht, JG. 66, 10/99, vom 11. März 1999, 193-200

DIW (6/1999): $\mathrm{CO}_{2}$-Emissionen in Deutschland: Weiterhin vom Zielpfad entfernt; in: DIW-Wochenbericht, JG. 66, 6/99, vom 11. Feb. 1999, 23-133

Bartholomai, Bernd (2000): Energieeinsparung im Wohnungsbestand - Investitionen müssen verstärkt werden; in: DIW-Wochenbericht, JG. 67, 31/2000, vom 3. August 2000, 499-507

Bellebaum, Alfred, Herbert Schaaff, Karl Georg Zinn (1999) (Hg.): Ökonomie und Glück. Beiträge zu einer Wirtschaftslehre des guten Lebens. Opladen/Wiesbaden

Binswanger, H.C., u.a. (1983): Arbeit ohne Umweltzerstörung. Strategien einer neuen Wirtschaftspolitik. Eine Publikation des „Bundes für Umwelt und Naturschutz Deutschland e.V.“, Frankfurt/M.

Blazejcak, J., D. Edler (1997): Tendenzen der umweltschutzinduzierten Beschäftigung in Deutschland; in: DIW-Wochenbericht, JG. 64, 9/1997, vom 27. Feb. 1997, 157-162

Blien, Michael, Michael Hauff, Jens Hornbach (2000): Beschäftigungseffekte von Umwelttechnik und umweltorientierten Dienstleistungen in Deutschland; in: Mitteilungen aus der Arbeitsmarkt- und Berufsforschung, JG. 33, H 1/2000, 126-135

Bode, Thilo (2000): Die Regierung hat kein zukunftsweisendes Umweltkonzept. Die Risiken neuer Techniken müssen öffentlich diskutiert werden/Greenpeace-Chef Thilo Bode im F.A.Z.-Gespräch; in: Franfurter Allgemeine Zeitung, Nr. 202, vom 31. Aug. 2000, 19

Brösse, Ulrich (1995): Ökonomisches Verhalten als bester Weg zu einer nachhaltigen Umweltverbesserung; in: Sigurd Martin Daecke: Ökonomie contra Ökologie? 78-85

Brundtland-Report (1987): The World Commission on Environment and Development, (Hg.), Our Common Future. Oxford

Daecke, Sigurd Martin (1995) (Hg.): Ökonomie contra Ökologie? Wirtschaftsethische Beiträge zu Umweltfragen. Stuttgart/Weimar 
Dyckhoff, Harald (2000): Umweltmanagement. Zehn Lektionen in umweltorientierter Unternehmensführung. Berlin u.a.

Fues, Thomas (1996): Humankapital und Naturvermögen. Der neue Weltbank-Index für Wohlstand und Nachhaltigkeit; in: E \& Z (Entwicklung und Zusammenarbeit), JG. 37, H 11, 301-303

Gocht, Werner (1995): Umwelt und Entwicklung: Armut als Ursache für Umweltschäden; in: Sigurd Martin Daecke: Ökonomie contra Ökologie? 86-93

Harborth, Hans-Jürgen (1999): Nachhaltiges Wirtschaften: Ressourceneffizienz und menschliche Genügsamkeit als neue Leitbilder?; in: Alfred Bellebaum u.a.: Ökonomie und Glück 170-192

Hans-Böckler-Stiftung (HBS) (2000) (Hg.): Wege in eine nachhaltige Zukunft. Ergebnisse aus dem Verbundprojekt Arbeit und Ökologie, Düsseldorf

Hillebrand, Bernhard, Klaus Löbbe (2000): Nachhaltige Entwicklung in Deutschland - Ausgewählte Problemfelder und Lösungsansätze, Untersuchungen des Rheinisch-Westfälischen Instituts für Wirtschaftsforschung, H 26, Essen

Hintemann, Ralph (2000): Die Diffusion umweltfreundlicher und hochwertiger Gebrauchsgüter dargestellt am Beispiel des 3-Liter-Autos (wirtschafts- und sozialwiss. Diss., Aachen; eingereicht)

Human Development (1999): Human Development Report 1999, United Nations Development Program (Hg.) New York-Oxford

Mayring, Philipp (1999): Lehren der neueren Psychologie für die Ökonomie: Welchen Stellenwert hat die Ökonomie für das menschliche Lebensglück wirklich?; in: Alfred Bellebaum, (1999): 157-169

Meadows, Donella H. u.a. (1972): The Limits to Growth, New York

Menschliche Entwicklung (2000): Bericht über die menschliche Entwicklung 2000, Deutsche Gesellschaft für die Vereinten Nationen, (Hg.), Bonn

$\mathrm{Ng}$, Yew-Kwang (2000): Why do economists overestimate the costs of public spending?; in: Royal Economic Society Newsletter,Nr. 110, Juli 2000, 5-7

Priewe, Jan (2000): Von Rom nach Wuppertal? Auf der Suche nach den ökologischen Grenzen des Wachstums. Ökologische Leitplanken für eine nachhaltige Entwicklung; in: Fritz Helmedag, Norbert Reuter (Hg.): Der Wohlstand der Personen. Marburg, 421-441

Sachs, Wolfgang (2000a): Effizienz als Destruktivkraft. Ökologische Folgen der Globalisierung; in: Blätter für deutsche und internationale Politik, JG.45, August 2000, 976-985

Sachs, Wolfgang (2000b): Wie zukunftsfähig ist Globalisierung, Wuppertal Papers, Nr. 99, Jan.2000; www.wupperinst.org/publikationen/wp99.pdf.

Schaaff, Herbert (1999): Zum Zusammenhang von ökonomischer Entwicklung, Wohlstandsentwicklung und menschlichem Wohlbefinden .- Historische Lehren für eine „ökologische Glücksökonomie"; in: Alfred Bellebaum: Ökonomie und Glück, 23-58

Schettkat, R. (1997): Die Intedependenz von Produkt- und Arbeitsmärkten. Die Wirtschafts- und Beschäftigungsentwicklung der Industrieländer aus der Produktmarktperspektive; in: Mitteilungen aus der Arbeitsmarkt- und Berufsforschung, Jg. 30, 4/2000, 721-731

Serageldin, Ismail (1996): Sustainability and the Wealth of Nations, Washington: Word Bank, Draft for Discussion, $2^{\text {nd }}$ edit., März 1996

Überleben sichern (1980): Das Überleben sichern. Gemeinsame Interessen der Industrie- und Entwicklungsländer. Bericht der Nord-Süd-Kommission. Mit einer Einleitung des Vorsitzenden Willy Brandt, Köln

Verbundprojekt (2000) Deutsches Institut für Wirtschaftsforschung; Wuppertal Institut für Klima, Umwelt, Energie; Wissenschaftszentrum Berlin für Sozialforschung, Arbeit und Ökologie: Projektabschlussbericht. Hg. v. der Hans-Böckler-Stiftung, Düsseldorf

Vinod, Thomas u.a. (2000): The Quality of Growth. Oxford/New York

Ziesing, Hans-Joachim (2000): Klimaschutzpolitik auf dem richtigen Weg, aber weitere Schritte unabdingbar; in: DIW-Wochenbericht, JG. 67, Nr. 32-33/2000, vom 10. Aug. 2000, 517-525

Zinn, Karl Georg (1999): Massenarbeitslosigkeit und Massenwohlstand. Das Janusgesicht unseres Kapitalismus zum Jahrhundertende und die Optionen der Beschäftigungspolitik; in: Aus Politik und Zeitgeschichte. Beilage zur Wochenzeitung „Das Parlament“, B 14-15/99, vom 2. April 1999, 3 13 
Zinn, Karl Georg (1998): Die Langfristperspektive der Keynesschen Wirtschaftstheorie; in: Das Wirtschaftsstudium (WISU), JG. 27, H 8-9/1998, 926-935

Zinn, Karl Georg (1997): Jenseits der Marktmythen. Wirtschaftskrisen: Ursachen und Auswege, Hamburg

Zinn, Karl Georg (1995): Wie umweltverträglich sind unsere Bedürfnisse? Zu den anthropologischen Grundlagen von Wirtschaftswachstum und Umweltzerstörung; in: Sigurd Martin Daecke: Ökonomie contra Ökologie? 31-62

Zinn, Karl Georg (1980): Die Selbstzerstörung der Wachstumsgesellschaft. Politisches Handeln im ökonomischen System. Reinbek bei Hamburg

Anschrift des Verfassers:

Prof. Dr. Karl Georg Zinn

Lehrstuhl für Volkswirtschaftslehre

Rheinisch-Westfälische Technische Hochschule

Templergraben 55

D-52062 Aachen

Schlagwörter: Arbeit, Beschäftigung, Nachhaltigkeit, Strukturwandel 